\title{
Aspects of Surgical Treatment of Rectal Cancer
}

\author{
Shaposhnikov Veniamin Ivanovich* \\ Professor of surgical diseases, Kuban Medical Institute, Russia
}

*Corresponding author: Shaposhnikov Veniamin Ivanovich, Professor of surgical diseases, Kuban Medical Institute, Russia.

Received Date: October 25, 2018

Published Date: December 18, 2018

\begin{abstract}
The author has perfected the technique of anterior resection of the rectum, which can reduce the risk of development, such as postoperative peritonitis and cicatricial stenos is of anastomosis. This is achieved by dipping the anastomosis in the lumen of the stump straight Kisi and the use of elastic rings to give it rigidity. The technique has been successfully applied at 25 patients.

Keywords: Rectal cancer anterior resection; Invigilated anastomosis

The purpose of the study: To determine the effectiveness of anterior resection of rectal cancer with the use of invaginacionnogo anastomosis.
\end{abstract}

\section{Introduction}

Rectal cancer is $4-6 \%$ of all malignant tumors in humans (8-9 places). Among malignant lesions of rectum, it is $80 \%$. Rectal cancer is more common in the age group 40-60 years and mostly in men (3:2). Patients with stage III-IV process constitute the overwhelming majority-81.2\%. Initial form (stage I), in which surgical treatment guarantees almost $100 \%$ 5-year survival rates are only operations $3.9 \%[1,2]$. For staging of timorous process and identify clinical prediction, widely used classification C.E. Dukes. It which into account two main pathological indication is the depth of germination of tumors in the intestinal wall and the presence of metastases in the lymph nodes. So, stage and to cases in which the growth of the tumor is limited to the wall of the rectum; in this stage the tumor spreads to surrounding tissues, but metastases in the lymph nodes do not exist; stage $\mathrm{c}$ is swelling with any degree of local dissemination with the presence of regional lymph node metastasis. The most widespread and recognized international classification of colorectal cancer, the proposed International Anticancer Union using characters TNM (Tumor, Metastases, Nodules). Clinical manifestations colorectal cancer depends on the stage of the disease, the nature of the growth of the tumor and its location $[1,3,4]$. The most frequent and constant symptom of colorectal cancer is bleeding. It occurs in both the early and later stages and in $75-90 \%$ of patients. It is often confused with hemorrhoids [5,6]. The second most symptoms are different types of bowel function disorders. Pain sensations appear when local spread of the tumor, especially as it moves to the surrounding organs and tissues $[7,8]$.

Violation of the general condition of patients (weakness, fatigue, anemia, weight loss, pallor of the skin) due to daily blood loss as well as neoplastic intoxication and observed in the later stages of the disease $[1,4]$. The doctor has pretty great opportunities for timely diagnosis of colorectal cancer at early stages should only have cancer suspicion. For this purpose, use the digital rectal, rektoskopiju, occult blood feces analysis, cytology reinstate [5,7]. Great importance to practical surgery is exploring ways of metastasis in cancer this localization. The appearance of metastases, the more remote, the main reason for neoperabelnosti tumors. Rectum in lymph flow is divided into upper and lower parts. The border between them lies at a height of 5-6 cm from zadneprohodnogo holes. In cancer, located within the channel anus Metastasis occurs on the back of its surface, as well as in the sacral, podchrevnye, inguinal lymph nodes. Metastasis of the tumor, which is located at 5-6 cm above the anus, occurs on the upper prjamokishechnoj and lower bryzheechnoj arteries. And through the lymph paths in parakavalnye and aortic lymph nodes and the liver $[1,2,8]$. Metastases in distant organs develop as a result of cancer of the lymph paths or elements in the blood stream. Surgical method is the leading in the treatment of colorectal cancer $[4,9,10]$. The main radical operations include: 1) abdominal-perinea 
extirpation of rectum by Kenju-Myles (at racah to 6-8 $\mathrm{cm}$ above the anus with odnostvolnoj colostomy in the left iliac region); 2) abdominal resection of rectum with revealing the sigmoid colon and the preservation of the sphincter (when the tumor above 6-8 $\mathrm{cm}$ from the anus); 3) anterior resection of rectum with restoring its continuity by anastomosis of type "end-to-end" (when the tumor on 7-8 cm above the anus); 4) resection of rectum and left half of the colon without restoring its continuity Hartmann method (in case of cancer or supraampuljarnogo rektosigmovidnogo from). In recent years, began to apply minimal invasive cancer removal technique that body [8].

Operational interventions in the rectum are classified as the most traumatic. The choice of the type of surgery colorectal cancer is very complicated and depends on many factors: tumor, macro-and microscopic its structure, the degree of local spread of tumor process, its metastasis and general condition of the patient. Clinical-anatomical and morphological studies have shown that to comply with the principles of the radicalism of surgery on the body enough to retreat from the lower pole of the tumor on 2-5 $\mathrm{cm}$ and from the upper pole to $12-15 \mathrm{~cm}$. Therefore, one of the key factors influencing the choice of the method of operation is the level of the lower pole of the tumor. A common view that the most radical operation of rectal cancer is abdominal-perinea extirpation of rectum, now hardly acceptable as with oncology positions and in terms of possible follow-up social and labor rehabilitation. According to the Research Institute of proctology in more than $68 \%$ of patients with cancer of the colon tumor is outside the zone of the obdurate apparatus of the rectum and, consequently, there are preconditions for its conservation with radicalism. When the abdominal area proctectomy, with revealing the colon in the analnyj channel on Soave, the rectum is removed, but the analnyj Canal and Parietal sphincter persisted. After being relegated the colon on the crotch is formed koloanalnyj anastomosis. This technique is popular with surgeons-oncologists. Its main drawback is the possibility of development of cicatricial stenosis in the area of the anus, devoid of the dentate line mucosa. When you run the front proctectomy from the bottom edge of the tumor needs to back off to not less than $5 \mathrm{~cm}$, and from the top of the Pole-on 12-15 cm [2,10].

After removal of the affected segment of straight line and part of the sigmoid colon is formed colorectal anastomosis type end-to-end. When "high" rezekcijah front usually formed double-row or single row anastomosis A traumatic needle, while "low" using a surgical stapling instrument. It was felt that, in addition to other advantages it profoundly enough is the preservation of zamykatelnogo apparatus of the rectum, no violation of the reproductive system and urinary disorders. However, this operation has one serious drawback is the technical difficulty of imposition mezhkishechnogo anastomoza deep in the pelvis. That is why this type of operation in the anastomosis seams insolvency $15-20 \%$ of patients, and his spinal stenosis-1-3\% [1,2,9]. Anterior resection is contraindicated in patients with symptoms of intestinal obstruction. In this case, Hartmann operation is performed. The final amount and type of surgical intervention are determined after laparoscopy and a thorough audit of the abdominal organs. Used nizhnesredinnyj cut. It has been proven that during mobilization of colon and anastomosis under the influence of mechanical trauma happens her thrombosis of blood vessels. This dramatically increases the risk of insolvency of seams anastomosis. Given all these negative factors front resection, we improved this method by developing a method invaginacionnogo colorectal anastomosis, designed for prevention of inflammatory complications (The RF patent № 2088157 from 02.08.93).

\section{Materials and Methods}

A generally accepted method to the extent necessary mobilizes get with ligatures on the barrel bottom artery. Below the rectum tumor mobilize on 5-6 cm. Then produce resection mobilized departments of colorectal tumor in one block, with the hollow organs impermeability provide overlaid circular ligatures with leaving their ends. Make the end of the sigmoid to the cult. Impose a single row mezhkishechnyj anastomosis. For this purpose, take the elastic plastic ring with a wall thickness of $2-2,5 \mathrm{~mm}$ and a diameter of 30-50 $\mathrm{mm}$ outer contour has notches that slid on it imposed. Before entering the ring in the anastomosis to it tie nylon thread.
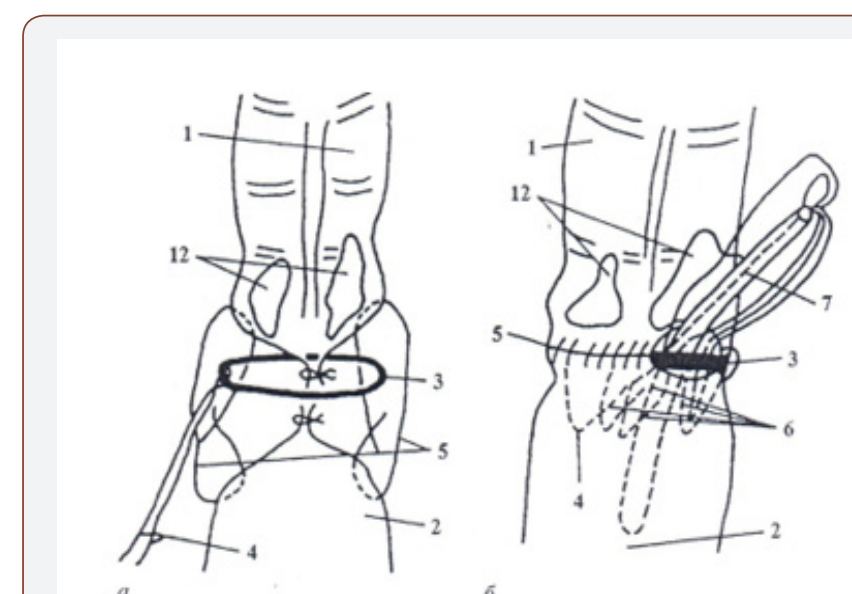

$a$

6
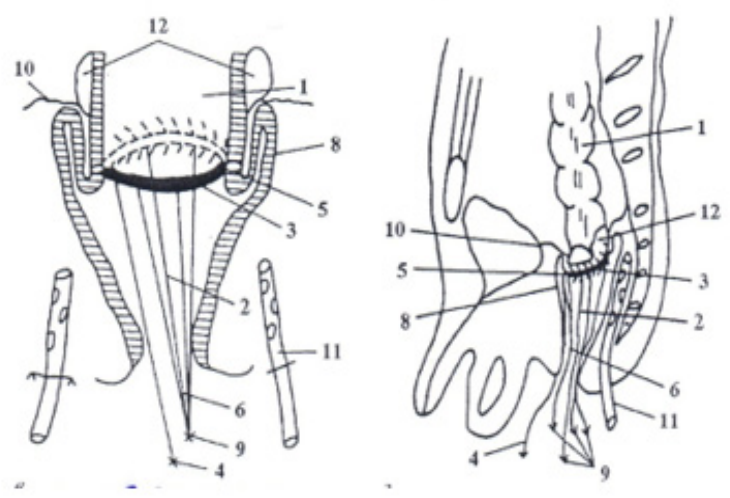

Figure 1: Reflected the scheme operation (a, b, c, d).

In Figure 1 (a, b, c, d) reflected the scheme operation, where 1-stump sigmoid colon, rectal stump-2, 3-a plastic ring, 4-nylon thread tied to the ring. Then, without opening the lumen of hollow organs begin to form the back-lip anastomosis using catgut threads \# 2-3 (5). In seam capture only by-muscular-the sub mucosal layer of the sigmoid colon and the sub mucosal muscle-direct. Then with all the left ends of the catgut strings (6) lock ring to the uzelkam formed the back lip of the anastomosis. Following this, cut threads 
(except the two laterals and one) and start forming the front lip of the anastomosis, with nodules have inside and they just fix the ring. When the front lip it temporarily leaves the window $20-25 \mathrm{~mm}$ diameter. Through this window into the abdominal cavity output ends of strands that are attached to the ring. Through a window cut the ends of the stumps of both hollow organs, and then through it same in rectal stump introduced tight probe (7), which then appears outside. By the end of the tube, which is located in the abdomen, attach yarn, bound to the ring. Traction for all threads are implementing invaginaciju anastomosis along with ring clearance in the rectal stump to a depth of 2-3 cm (8). Surface area covered with hollow organs die fat pendants (12). The ends of the filaments and the tube coming out of the anus, fasten the inner thighs (9). The pelvic peritoneum covers the anastomosis (10). In presakralnoe space zabrjushinno misleading perforated drainage tube with the two iliac areas (11).

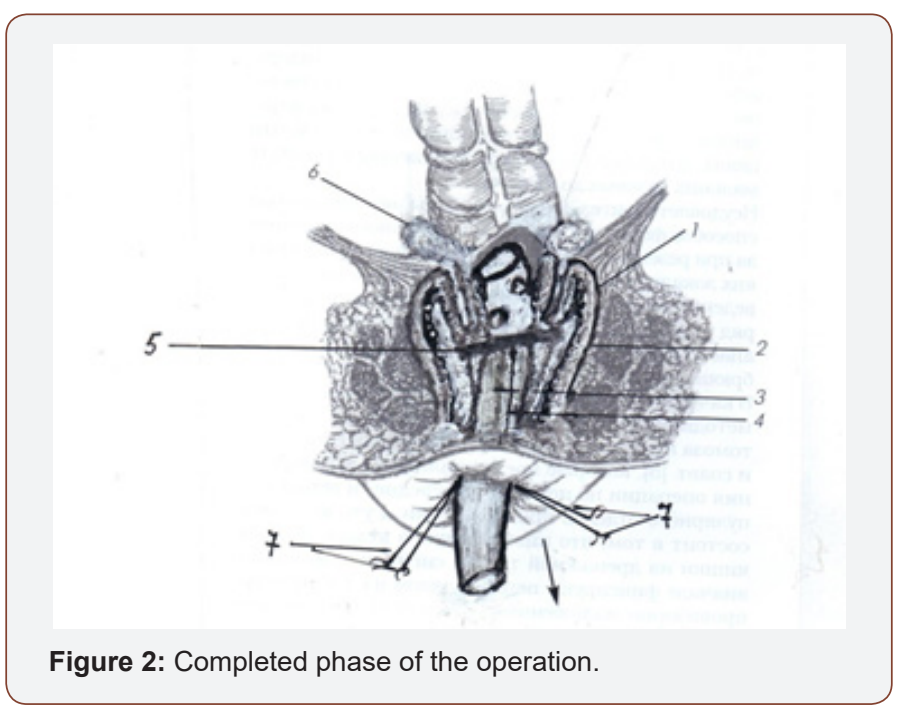

In Figure 2 submitted a completed phase of the operation, where 1-invaginirovannyj anastomosis, Division 2-wall of the rectum, 3-tube in the rectum, 4-nylon thread attached to a ring, 5-invaginirujushhee, 6-ring of fat trimmings, 7-thread locking ring derived through the anus and fortified skin of upper/third of thigh. The tube is removed through the 3-5 days, when depart Gaza, and ligatures them depart through the 8-10 days. The ring is removed by sheet metal loop creating for nylon yarn.

\section{Result}

The described method of anterior resection of the rectum was applied from 25 patients aged 53 to 72 years. The lower pole of the tumor was at a distance of $10-12 \mathrm{~cm}$ from the anus. Form tumor histology is adenocarcinoma. The length of the gut wall lesions from 6 to $8 \mathrm{~cm}$. During an operation found that the tumor is movable, ie will not germinate in the adjacent organs and tissues. These clinical data allowed performing radical surgery on the developed methodology. The operation was performed by single Brigade surgeons and 3-4 lasted hours. Both during and after the operation, complications were observed. Duration of hospitalization was 3-4 week. After that, patients are transferred to the Oncology Center, where they received a course of radiation therapy. Died 1 patient (4\%) at the age of 72 years of acute cardiovascular insufficiency.

\section{Discussion}

If the patient testimony to the fulfillment of the primary anterior resection of the rectum, you can use a technique of submersible anastomosis with submersible elastic ring. This anastomosis prevents the development of peritonitis due to the eruption of joints formed by soustja, as it is in the lumen of the stump of the rectum and, if it even happens, hollow organs impermeability is not broken. Elastic ring which is located in the gleam of anastomosis, warns his umbilicus constriction within $6-7$ days. Clinical observations have shown that, during this time comes spajanie anastomosis with the surrounding tissue of the pelvis and since rubber rings stricture does not develop.

\section{Conclusion}

The developed method of front proctectomy allows is significantly reduce the risk of postoperative peritonitis due to insolvency of the seams as the anastomosis, anastomosis plunges into the lumen of the rectal stump. Applied ring performs well as a preventive role in the development of anastomotic stricture of anastomosis. This technique makes it way to the anterior resection more secure.

\section{Acknowledgment}

None.

\section{Conflict of Interest}

No conflict of interest.

\section{References}

1. Fedorov VD, Sparrows, VI Rivkin GI (1994) Clinical operational Coloproctology. -M: medicine, pp.335s.

2. Armstrong DN, Ambrose WI, Schertzer ME (2002) Harmonic scalpel hemorrhoidectomy. Dis. Colon Rec-tum 45: 354-359.

3. Aleksandrov VB (1977) Rectal cancer. M Meditsina, pp.20.

4. Aminev AM (1971) Guide to proctology. In: Kuibyshev (Ed), vol. 2.

5. Bayer I, Myslovaty B, Picovsky BM (1996) Rubber band ligation of hemorrhoids. Convenient and economic treatment. J. Clin. Gastroenterology 23(1): 50-52.

6. Trudel JL (1994) Ferguson's method of closed hemorrhoidectomy. AnnChir 48(6): 561-564.

7. Gawenda M, Walter M (1996) Surgical therapy of advanced hemorrhoidal disease: is an ambulatory surgery intervention possible? Chirurg 67(9): 940-943.

8. Морис Е, Арегви, Джонатан М, Сакиера А (1999) Малоинвазивная колопроктология. М.: Медицина, pp.64c.

9. Drobni SH (1983) Bowel Surgery. Budapest.

10. Red AN (1968) Atlas operations on rectum and large intestines. Moscow, pp.335. 